\title{
CHANGE DETECTION OF POLARIMETRIC SAR DATA FOR MONITORING OF AGRICULTURAL AREAS
}

\author{
Neelanshi Varia ${ }^{1, *}$, Nidhi Davawala ${ }^{1}$, Sanid Chirakkal ${ }^{2}$, Dipanwita Haldar ${ }^{3}$, Ranendu Ghosh ${ }^{1}$, Deepak Putrevu ${ }^{2}$ \\ ${ }^{1}$ Dhirubhai Ambani Institute of Information and Communication Technology, Gandhinagar, India - \\ (neelanshiV2, davawalanidhi)@gmail.com,ranendu ghosh@daiict.ac.in \\ ${ }^{2}$ Microwave Techniques Development Division, Space Applications Centre, ISRO, Ahmedabad, India, (sanid, dputrevu)@sac.isro.gov.in \\ ${ }^{3}$ Indian Institute of Remote Sensing, Dehradun, India, dipanwita@iirs.gov.in
}

\section{Commission V, SS: Natural Resources Management}

KEY WORDS: Change Detection, Test statistic, Hypothesis testing, Polarimetric SAR

\begin{abstract}
:
Change detection is important to understand the patterns of transition in multi-temporal SAR acquisitions over same geographical areas. In this work, we implemented a test statistic on covariance matrices for change detection. The RADARSAT-2 data spanning the agricultural land of Central Hisar Farm in Haryana, India was used. Hypothesis testing on test-statistic was done by a pre-decided significance level. A change map was plotted and the areas with 'change' and 'no change' were determined. Analysis of changing trends of different crop lifecycles is done. This study is useful in making important agricultural crop predictions.
\end{abstract}

\section{INTRODUCTION}

\subsection{General introduction}

Change detection in SAR images has become a crucial part of recent works in remote sensing. Various approaches ranging from the algebraic methods of image differencing and ratio [1] to the supervised methods of classification [2] and to the more recent unsupervised methods based on statistical and probabilistic approaches of test statistic [3][4] for change detection can be found in the literature. The test statistic has proven to be a very simple and efficient method.

\subsection{Our implementation}

In this paper, we implemented hypotheses testing on a test statistic for change detection in SAR images of an agricultural area. Agricultural land monitoring is an important field of research in which change detection plays a major role. The results of the test statistic are used to generate a change map which identifies the areas where changes have occurred over a span of time.

\section{STUDY AREA}

The Central State Farm of Hisar in Haryana, India was chosen as the study area owing to its large and demarcated farms in which a wide variety of crops are cultivated. The area lies between $29^{\circ} 11^{\prime}-29^{\circ} 20^{\prime} \mathrm{N}$ latitudes and $75^{\circ} 36^{\prime}-75^{\circ} 45^{\prime}$ E longitudes. The SAR acquisitions of this area are taken by a space borne satellite RADARSAT-2. The SAR images of datesDecember 14, 2011 and March 19, 2011 are used for our study. The SAR imagery obtained by RADARSAT-2 captures a vast agricultural land in the state of Haryana in India.

\footnotetext{
* Corresponding author
} 


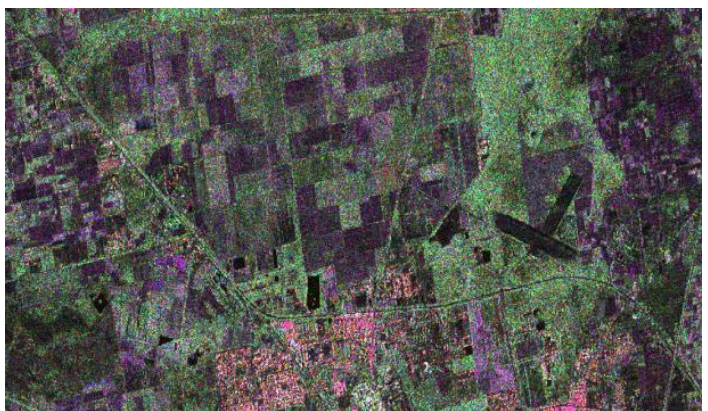

Figure 1: Composite of $\mathrm{HH}, \mathrm{HV}$ and VV bands of Hisar Farm

The entire land is full of patches of farms where a variety of crops are cultivated. The crops of wheat, mustard, gram, palak, cotton, arhar are most rampant in these areas. Scattered amongst the farms are some pockets of villages as well as urban areas, some fallow land, water bodies and regions of sand dunes. One of the key farms under observation is the Hisar Central farm which has well demarcated fields and a variety of crops. Ground truth information corresponding to the observed dates of acquisitions was collected for further analysis.

\section{METHODOLOGY}

\subsection{Overview}

For change detection, the method of hypothesis testing on a test statistic was implemented. A log likelihood based test statistic was used as a measure of equality of two distinct covariance matrices (C3). C3 is a $3 \times 3$ Hermitian matrix with a complex Wishart distribution for every pixel.

\subsection{Steps of implementation}

3.2.1 Log-test: To determine the equality of two of these covariance matrices, $\mathrm{A}$ and $\mathrm{B}$, the logarithm of test statistic $\mathrm{T}$ is defined as:

$\ln T=2 p \ln 2+\ln |A|+\ln |B|-2 \ln |A+B|$

For covariance matrix data, $\mathrm{p}=3$ and for $\mathrm{HH}, \mathrm{HV}$, or VV data, $\mathrm{p}=1$.
3.2.2 Hypothesis definition: The hypotheses are -

$\begin{cases}H 0, & \Sigma_{a}=\Sigma_{b} \\ H 1, & \Sigma_{a} \neq \Sigma_{b}\end{cases}$

The null hypothesis (H0) assumes that the covariance matrices $\mathrm{A}$ and $\mathrm{B}$ are equal while the alternate hypothesis (H1) assumes that they are different. Thus, H0 corresponds to 'no change' while H1 corresponds to 'change'.

3.2.3 Hypothesis testing: A hypothesis testing is done on the test statistic using the following expression as the threshold,

$P\left\{\chi^{2}\left(p^{2}\right) \leq z\right\}+\omega\left[P\left\{\chi^{2}\left(p^{2}+4\right) \leq z\right\}-\right.$ $\left.P\left\{\chi^{2}\left(p^{2}\right) \leq z\right\}\right]$

Here, $\mathrm{z}$ is the significance level while $\omega$ is function of parameters $p$.

3.2.4 Thresholding: For each pixel, the null hypothesis was rejected if the value of ' $2 \rho \operatorname{lnT}$ ' is less than the above threshold value. Where $\rho$ and $\omega$ is,

$$
\begin{aligned}
& \rho=1-\frac{2 p^{2}-1}{6 p}\left(\frac{1}{n}+\frac{1}{m}-\frac{1}{n+m}\right) \\
& \omega=-\frac{p^{2}}{4}\left(1-\frac{1}{p}\right)^{2}+\frac{p^{2}\left(p^{2}-1\right)}{24 \rho^{2}}\left(\frac{1}{n^{2}}+\frac{1}{m^{2}}-\right. \\
& \left.\frac{1}{(n+m)^{2}}\right)
\end{aligned}
$$

The $\chi^{2}$ distribution in eq. (2) is given by,

$$
P\left(\chi^{2} \leq a\right)=\int_{0}^{a\left(\frac{1}{2}\right)^{\frac{k}{2}} x^{\frac{k}{2}-1} e^{-\alpha x}} \frac{\Gamma\left(\frac{\mathrm{k}}{2}\right)}{} d x
$$

Where, $\Gamma(t)=\int_{0}^{\infty} x^{t-1} e^{-x} d x$ 
Here $\mathrm{n}$ and $\mathrm{m}$ is the equivalent number of looks of the two covariance matrices respectively.

3.2.5 Change map generation: Using this, a change map is generated in which the darker regions depict 'change areas' and the brighter regions depict 'no change areas'. The test statistic was applied for 3 cases

1. Consider only the Svv values for all the resolution cells

2. In the $3 \times 3$ covariance matrix (C3) the nondiagonal values are set to 0

3. The entire $3 \times 3$ complex covariance matrix (C3) is considered

\section{RESULTS AND DISCUSSION}

Fig. 2 shows $\ln \mathrm{T}$ for all the 3 cases. Darker regions correspond to more changes whereas the brighter regions correspond to lesser amount of changes i.e. higher the value of $\ln \mathrm{T}$, lesser will be the change.

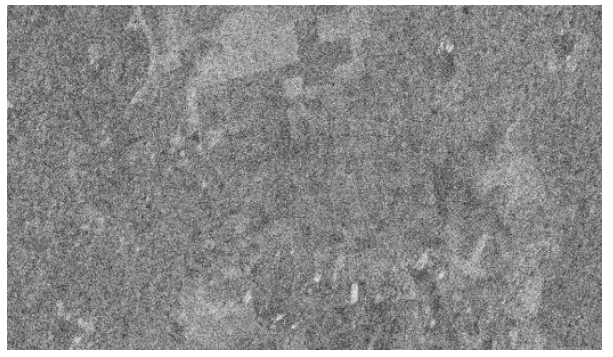

(a)

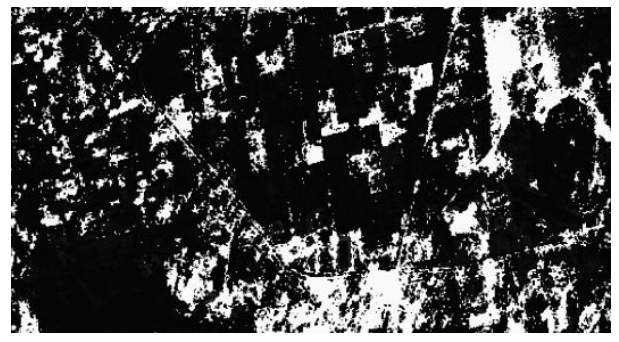

(b)

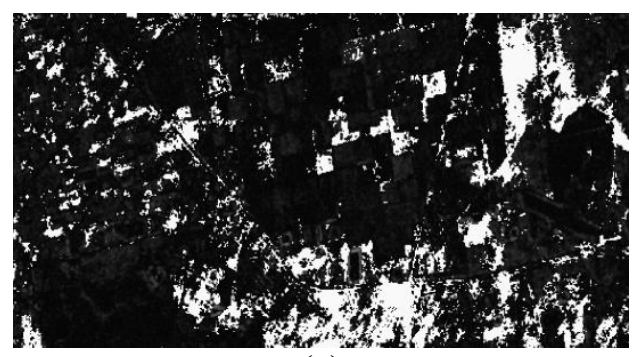

(c)

Figure 2: Test statistic output on different cases. (a) Only VV channel (b) Only diagonal elements of C3 (c) Using full

C3

It can be observed from fig. 2 (a) that the value of ' $I n T$ ' is similar for all the pixels, i.e. the VV channel alone is not able to detect change very efficiently. Whereas, in fig.2 (b), the results are much better than case 1 . Here, darker and brighter regions can be seen distinctly. In fig.2 (c) the regions contain lesser amount of noise (i.e. scattered pixels). In the case 2, majority values of $\ln \mathrm{T}$ obtained denote either complete change or no change i.e. black and white pixels. While in case 3 , the values of $\ln T$ obtained show regions with changes that were not detected earlier (grey areas which were white in case 2). Thus, case 3 gives the best output of change detection.

Hence for further steps, we considered case 3. We apply a threshold on images with $1 \%$ and $5 \%$ significance level and reject the null hypothesis of the pixels for which the value of ' $2 \rho \ln T$ ' is less than the threshold. Fig. 3 Clearly shows the output for change detection on $1 \%$ significance level (a) has lesser areas showing change whereas (b) has larger areas depicting change.

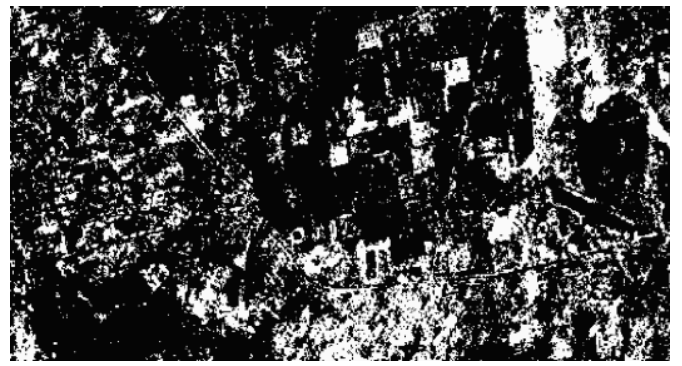

(a) 


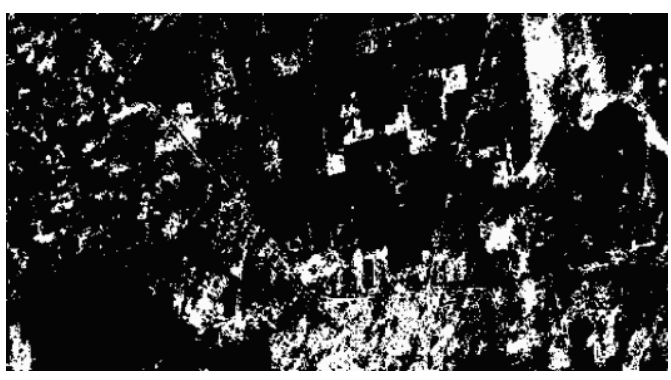

(b)

Figure 3: Rejection of null hypothesis based on various significance levels (a) $1 \%$ (b) $5 \%$

From the ground truth, it can be observed that wheat area changes a lot from March to December. The fully grown wheat areas are now either fallow land or recently sown wheat patches. Similarly, a lot of areas having mustard are changed. Whereas the percentage change of areas where there is gram is minimal. The urban areas can also be seen in white patches and hence represent very less change. Fig. 4 shows probability of three crops, urban area and fallow land changing from March to December.

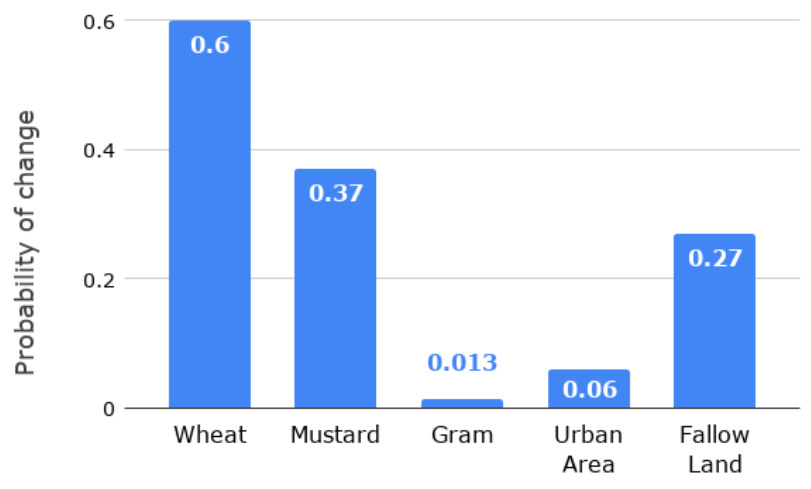

Figure 4: Probability of change for different classes

\section{CONCLUSION}

Using test statistic, we generated a change map between two scenes of Hisar farm in Haryana. We analyzed the results by using 3 cases of varying SAR information and performing hypothesis testing with different significance levels. Finally, we calculated the probability of change for each class. Further we plan to include a larger variety of crops and higher number of SAR acquisitions for more accurate time series analysis.

\section{REFERENCES}

[1] Rignot, E. J., \& Van Zyl, J. J. (1993). Change detection techniques for ERS-1 SAR data. IEEE Transactions on Geoscience and Remote sensing, 31(4), 896-906.

[2] Bruzzone, L., Prieto, D. F., \& Serpico, S. B. (1999). A neural-statistical approach to multitemporal and multisource remote-sensing image classification. IEEE Transactions on Geoscience and remote Sensing, 37(3), 1350-1359.

[3] Conradsen, K., Nielsen, A. A., Schou, J., \& Skriver, H. (2003). A test statistic in the complex Wishart distribution and its application to change detection in polarimetric SAR data. IEEE Transactions on Geoscience and Remote Sensing, 41(1), 4-19.

[4] Akbari, V., Anfinsen, S. N., Doulgeris, A. P., Eltoft, T., Moser, G., \& Serpico, S. B. (2016). Polarimetric SAR change detection with the complex Hotelling-Lawley trace statistic. IEEE Transactions on Geoscience and Remote Sensing, 54(7), 3953-3966. 